\title{
MODEL OF QUASI-LINEAR RHEODYNAMIC MEDIUM AND ITS SOLUTION
}

\author{
Akmal A. Mirzoev ${ }^{1}$, Ikhmat N. Khusanov ${ }^{2}$ \\ ${ }^{I}$ Department of "Projection of Engineering Communications, Build and Using" - Tashkent Architecture Construction \\ Institute, Tashkent City, 11, Navoi Street, Uzbekistan \\ ${ }^{2}$ Department of "Projection of Engineering Communications, Build And Using” - Tashkent Architecture Construction \\ Institute, Tashkent City, 11, Navoi Street, Uzbekistan
}

\begin{abstract}
This paper analyses the stable and unstable stress deformation states of elastic-viscous-inertial (retardation) model depending on the acting stress and rheological parameters of the medium. The introduced concepts: self-oscillatory deformation state, the soft mode of deformation, hard deformation mode, excitation coefficient of rheodynamcs system and others. For the initial conditions lying within the unstable, limit cycle the oscillatory deformation of rheodynamcs system is damping. For the initial conditions, located outside of this cycle, self-oscillatory deformation processes are set. Parameter $\alpha$, resulting in different values to the emergence and disappearance of self-oscillation in a medium, is called coefficient of stimulating rheodynamcs systems. However, an unlimited increase in the vibration strain requires unlimited admission of energy to the system. Therefore, in these cases, the approach taken under different $\tau$ is to be enough and take into account the higher-order terms.
\end{abstract}

Keywords: Deformable Medium, Linear Viscoelasticity, Viscous - Inert, A Rheodynamcs, A Quasi-Linear, SelfOscillatory, A Stable Equilibrium State

\section{NOMENCLATURE}

$\gamma \quad$ deformation

$\dot{\gamma} \quad$ deformation rate [1/s]

$\ddot{\gamma} \quad$ accelerated deformation $\left[1 / \mathrm{s}^{2}\right]$

$G \quad$ coefficient of elasticity of the medium $\left[\mathrm{N} / \mathrm{m}^{2}\right]$

$\mu \quad$ coefficient of dynamic viscosity of the medium $[\mathrm{Pa}$

$\mathrm{s}]$

$m_{\ell} \quad$ linear density of the medium $[\mathrm{kg} / \mathrm{m}]$

$\tau \quad$ shear stress $\left[\mathrm{N} / \mathrm{m}^{2}\right]$

$t \quad$ time [s]

$x \quad$ length $[\mathrm{m}]$

$\eta \quad$ small parameter

Kh Khusanovs number

$\alpha \quad$ a new variable

$\beta \quad$ a new variable

$\delta \quad$ a new variable

$\rho \quad$ a new variable

$g \quad$ a new function

$\Phi(\rho) \quad$ a new function

\section{INTRODUCTION}

The transition process is accompanied by the work of machines with hydraulic drives, determining changes in pressure patterns and working fluid rate, depending on the rheological properties and movement of the executive bodies of high-speed mechanisms is an urgent task. Increasing the speed and accuracy of modern hydraulic drive, where in most cases in the form of working fluids used non-Newtonian fluid, causing the need for in-depth study of the dynamics of the number of transport processes such substances liquids.

Lorenz E. N. (1963). was first pointed out that in the turbulent motions friction - turbulent viscosity, in contrast to the usual viscosity is determined by the transfer through the layers of a moving liquid momentum is not microscopic particles, and stirred the finite volume of the medium.

At the present stage of development of the mechanics of movements of one and two-phase media, the establishment of the laws of the transfer processes of physical substances, construction of mathematical models as the movement and interactions between the phases and to limit the flow of the surface, as well as the development of methods of solution were in great contribution work of the following scientists: Jeffreys, H. (1926), Oldroyd, J. G. (1950), Eirich F. R. (1956-1970), W. Prager, C. Truesdell, Coleman B. D., and Noll W., (1961), Landau, L. D. and Lifshitz, E. M. (1987),R.I. Nigmatulin et al ( 1990, 1996), C. Kuehn. (2015), and others.

Schlichting (1979), L.G. Loitsyansky (1987), and others argue that for the molar internal transport processes the main characteristic value should be a dynamic flow rate.

Problem of molar transfer in the turbulent motion of the liquid and gas; the problem of migration of the dispersed phase in the dispersion medium moving at low flow rates; the problem of formation, as well as the destruction of the structure (core) flow with the motion- visco-plastic media - 
the three current problems of hydro-aerodynamics merge into one problem, the establishment of the problem more adequately describes the regularity of the internal molar transfer process in flows, which means the creation of more adequately describing the rheological models.

During this period of the development of science, the problem of internal transfer processes in the flow of liquids and gases has become a central issue of modern mechanics, physics and chemistry. The development of a large number of basic and applied fields of science is related to the development of understanding and theoretical description of the internal processes of the molar transfer of various kinds of substances in single and multiphase flow.

Therefore, there is need to find a new approach to modeling of the internal process of the molar transfer of fluid media in order to develop a mathematical model of the problem, taking into account the mechanism of transfers, more adequately describing the process and making it possible to improve knowledge in this field.

Since, in many practical situations, the evolution of the internal molecular and molar processes do not lend themselves directly to detailed mathematical modeling, it is important to develop a theory of internal processes that make it possible phenomenological to link the characteristics of the external action and system response with internal processes taking place in the system.

\subsection{Model of Viscous - Inert Deformable Medium}

In connection with the above stated, Khusanov I.N. in his work proposed a model of deformable inert medium, determined the accelerated deformation of the body relative to the coordinate system, which allows establishing the law of deformation inertia of the deformable body.

In which the body retains its deformed state, or even change it as long as it is not forced to change under the influence of external or internal stresses.

By analogy with the generalization of the theory of elastic deformation of deformable bodies and strain rate in a viscous fluid theory, we introduce the tensor components of accelerated deformation corresponding spatial change of acceleration.

Since in the deformation of the various media the difference in the properties and characteristics of their behaviour is clearly seen, the mentioning on the rheological equations, we talk about the relationship between strain rate, accelerated strain and stresses.

In I.N. Khusanov's work (1990), he introduced the accelerated strain tensor components corresponding to the spatial variation of the components of acceleration, in the form $\ddot{\gamma}_{i j}=\frac{\partial W_{i}}{\partial x_{j}}$ and set the law on which the product of the mass per unit length $\boldsymbol{m}_{\ell}$ and the tensor of accelerated strain $\ddot{\gamma}_{i j}$ directly proportional to the stress tensor $\tau_{i j}$ i.e.

$$
\tau_{i j}=m_{\ell} \ddot{\gamma}_{i j}
$$

\section{MODEL OF QUASI-LINEAR RHEODYNAM- ICS MEDIUM}

In the above processes, the working fluid is subjected to repeated acceleration and stopping. These processes are characterized with high-speed and accelerated changes in hydrodynamic and rheological parameters of liquids. Furthermore, highly viscous oil additives of oil products, clay and other concentrated solutions, polymer melts, whipped cream and ointments are many examples of rheological complex fluids. In flow of such fluids such effects, which have no analogues in the flows of Newtonian liquids, are observed. Such viscoelastic medium are mentioned, for example, in works of Butenin, N.V., et al. 1987; Larson, R.G. 1999; Barnes, H.A., et al. 2000; Kapitaniak T., 2000; Wiggins, S., 2003; Seydel R., 2010; Kuehn. C., 2015; Christova, I.C., and Christov, C.I., 2016.

In this paper, we study some of the properties of fluids obeying one of rheodynamcs models, proposed in (Khusanov, I.N., 1990; Seydametova, Z.S., 1990; Khusanov, I.N., and Mirzoev, A.A., 2015) as a quasi-linear rheodynamcs system in Figure 1, the stress-strain state, which is represented in the form of:

$$
m_{\ell} \ddot{\gamma}_{i j}+\mu \dot{\gamma}_{i j}+G \gamma_{i j}=\eta \tau_{i j}\left(\gamma_{i j}, \dot{\gamma}_{i j}\right)
$$

where $m_{\ell}, \mu, G$ - the constant coefficients; $\tau_{i j}\left(\gamma_{i j}, \dot{\gamma}_{i j}\right)$ non-linear function of stress, depending on component of strain $\left(\gamma_{i j}\right)$ and strain rate $\left(\dot{\gamma}_{i j}\right) ; \eta$ - small parameter. When $\eta=0$, the equation (1) becomes linear, when $\eta \neq 0$, but quite small, rheodynamcs system is close to linear and will therefore meet the definition of (Butenin, N.V., et al. 1987; Wiggins, S., 2003) autonomous system.

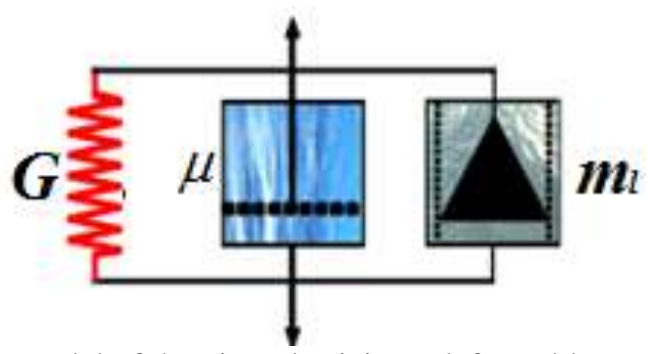

Fig. 1 Model of the viscoelasticinert deformable medium

\subsection{The Quasi-Linear Rheodynamic Equation}

Without going into the difference between the strain tensor components, rate and acceleration of strain and stress, equation (1) can be written as: 


$$
m_{\ell} \ddot{\gamma}+\mu \dot{\gamma}+G \gamma=\tau
$$

where $m_{\ell}$ - the linear density of the medium, $\mu$ - coefficient of dynamic viscosity of the medium, $G$ - coefficient of elasticity of the medium, $\tau$-stress.

We assume that the stress is a function relative to deformation rate $\dot{\gamma}_{i j}=\dot{\gamma}_{0}+\dot{\gamma}$. Assuming $\dot{\gamma}_{0}>|\dot{\gamma}|$, we expand $\tau\left(\dot{\gamma}_{0}+\dot{\gamma}\right)$ into series with powers of $\dot{\gamma}$ :

$\tau\left(\dot{\gamma}_{0}+\dot{\gamma}\right)=\tau\left(\dot{\gamma}_{0}\right)+\tau^{\prime}\left(\dot{\gamma}_{0}\right) \dot{\gamma}+\frac{1}{2} \tau^{\prime \prime}\left(\dot{\gamma}_{0}\right) \dot{\gamma}^{2}+\frac{1}{6} \tau^{\prime \prime \prime}\left(\dot{\gamma}_{0}\right) \dot{\gamma}^{3}$

$+\frac{1}{24} \tau^{I V}\left(\dot{\gamma}_{0}\right) \dot{\gamma}^{4}+\frac{1}{120} \tau^{(V)}\left(\dot{\gamma}_{0}\right) \dot{\gamma}^{5}+\ldots \cdot$

By limiting the number of members up to six and averaging, members of the second and fourth derivatives are cancelled, and therefore the equation of strain-stress state of the medium can be written as:

$$
\begin{aligned}
& \ddot{\gamma}+\frac{\mu}{m_{\ell}} \dot{\gamma}+\frac{G}{m_{\ell}} \gamma=\frac{\tau\left(\dot{\gamma}_{0}\right)}{m_{\ell}}+\frac{\tau^{\prime}\left(\dot{\gamma}_{0}\right)}{m_{\ell}} \dot{\gamma}+ \\
& +\frac{\tau^{\prime \prime \prime}\left(\dot{\gamma}_{0}\right)}{6 m_{\ell}} \dot{\gamma}^{3}+\frac{\tau^{(V)}\left(\dot{\gamma}_{0}\right)}{120 m_{\ell}} \dot{\gamma}^{5} .
\end{aligned}
$$

\subsection{The solution of a Quasi-Linear Rheodynamic}

\section{Equation}

Let us introduce the notation the parameters $t=t_{\text {ret }\left(K h_{2}\right)} t_{1}$, where $t_{r e t\left(K h_{2}\right)}=\left(m_{\ell} / G\right)^{1 / 2}$ and $t_{r e t\left(K h_{1}\right)}=m_{\ell} / \mu$, then from (2) we have:

$$
\begin{aligned}
& \frac{d^{2} \gamma}{d t_{1}^{2}}+\gamma=-\frac{t_{r e t\left(K h_{2}\right)}}{t_{r e t\left(K h_{1}\right)}} \frac{d \gamma}{d t_{1}}+\frac{t_{r e t\left(K h_{2}\right)}^{2}}{m_{\ell}} \tau\left(\gamma_{0}\right)+ \\
& +\frac{t_{r e t\left(K h_{2}\right)} \tau^{\prime}\left(\dot{\gamma}_{0}\right)}{m_{\ell}} \frac{d \gamma}{d t_{1}}+\frac{\tau^{\prime \prime \prime}\left(\dot{\gamma}_{0}\right)}{6 m_{\ell} t_{r e t\left(K h_{2}\right)}}\left(\frac{d \gamma}{d t_{1}}\right)^{3}+ \\
& +\frac{\tau^{v}\left(\dot{\gamma}_{0}\right)}{120 m_{\ell} t_{r e t\left(K h_{2}\right)}^{3}}\left(\frac{d \gamma}{d t_{1}}\right)^{5} .
\end{aligned}
$$

By introducing a new variable in the form of $g=\gamma+\frac{\tau\left(\dot{\gamma}_{0}\right)}{m_{\ell}} t_{\text {ret( }\left(K h_{2}\right)}^{2}$, from (3), we obtain:

$$
\begin{aligned}
& \frac{d^{2} g}{d t_{1}^{2}}+g=\left(\frac{\tau^{\prime}\left(\dot{\gamma}_{0}\right)}{m_{\ell}}-\frac{1}{t_{r e t\left(K h_{1}\right)}}\right) t_{r e t\left(K h_{2}\right)} \frac{d g}{d t_{1}}+ \\
& +\frac{\tau^{\prime \prime \prime}\left(\dot{\gamma}_{0}\right)}{6 m_{\ell} t_{r e t\left(K h_{2}\right)}}\left(\frac{d g}{d t_{1}}\right)^{3}++\frac{\tau^{(v)}\left(\dot{\gamma}_{0}\right)}{120 m_{\ell} t_{r e t\left(K h_{q}\right)}^{3}}\left(\frac{d g}{d t_{1}}\right)^{5} .
\end{aligned}
$$

Assuming:

$$
\begin{aligned}
& \frac{\mu}{\sqrt{G m_{\ell}}}=\frac{t_{r e t\left(K h_{2}\right)}}{t_{r e t\left(K h_{1}\right)}}<1, \quad \frac{\tau^{\prime}\left(\dot{\gamma}_{0}\right)}{m_{\ell}} t_{r e t\left(K h_{2}\right)}<<1, \\
& \frac{\tau^{\prime \prime \prime}\left(\dot{\gamma}_{0}\right)}{m_{\ell} t_{r e t\left(K h_{2}\right)}}<<1, \frac{\tau^{V}\left(\dot{\gamma}_{0}\right)}{m_{\ell} t_{r e t\left(K h_{2}\right)}^{3}}<<.
\end{aligned}
$$

Let $\eta=\left(\mu t_{r e t\left(K h_{2}\right)}\right) / m_{\ell}$ be a small parameter characterizing the proximity of the system to a linear conservative. Introducing the notation:

$$
\begin{aligned}
& \alpha=-1+\left(\tau^{\prime}\left(\dot{\gamma}_{0}\right) t_{\text {ret }\left(K h_{1}\right)}\right) / m_{\ell}, \\
& \beta=\left(t_{\text {ret }\left(K h_{1}\right)} \tau^{\prime \prime \prime}\left(\dot{\gamma}_{0}\right)\right) /\left(6 t_{\text {ret }\left(K h_{2}\right)}^{2} m_{\ell}\right), \\
& \delta=\left(t_{\text {ret }\left(K h_{1}\right)} \tau^{(v)}\left(\dot{\gamma}_{0}\right)\right) /\left(120 t_{\text {ret }\left(K h_{2}\right)}^{4} m_{\ell}\right),
\end{aligned}
$$

and considering them in the equation of the stress-strain state of the medium (4), we obtain:

$$
\frac{d^{2} g}{d t_{1}^{2}}+g=\eta \alpha \frac{d g}{d t_{1}}++\eta \beta\left(\frac{d g}{d t_{1}}\right)^{3}+\eta \delta\left(\frac{d g}{d t_{1}}\right)^{5}
$$

In accordance with I.N. Khusanov (1990) the right side of the equation (5) can be written in the form

$$
\Phi(\rho)=\frac{1}{2} \rho\left(\alpha+\frac{3}{4} \beta \rho^{2}+\frac{5}{8} \delta \rho^{4}\right)
$$

Then the state of equilibrium of the medium, obeying the equation of stress-strain state (5), is found from the equality:

$$
\Phi(\rho)=\frac{1}{2} \rho\left(\alpha+\frac{3}{4} \beta \rho^{2}+\frac{5}{8} \delta \rho^{4}\right)
$$

\section{ANALYSIS OF THE SOLUTION OF EQUA- TION}

The root $\rho_{1}=0$ corresponds to a state of equilibrium rheodynamcs system. Since the derivative of the function $\Phi(\rho)$ is:

$$
\Phi^{\prime}(\rho)=\frac{1}{2}\left(\alpha+\frac{9}{4} \beta \rho^{2}+\frac{25}{8} \delta \rho^{4}\right)
$$

then the equilibrium state $\rho_{1}=0$ is stable if $-1+\left(\tau^{\prime}\left(\dot{\gamma}_{0}\right) t_{r e t\left(K h_{1}\right)}\right) / m_{\ell}<0, \quad$ is not stable if $-1+\left(\tau^{\prime}\left(\dot{\gamma}_{0}\right) t_{r e t\left(K h_{1}\right)}\right) / m_{\ell}>0$. Other states of equilibrium are found from the equation: 
$\alpha+\frac{3}{4} \beta \rho^{2}+\frac{5}{8} \delta \rho^{4}=0$

Since we are interested in only positive roots of the equation (7), we consider how these roots depend on the coordinate for fixed $\beta$ and $\delta$.

The case when $\quad \beta<0 \quad$ and $\delta<0$, i.e. $\left(\tau^{\prime \prime \prime}\left(\dot{\gamma}_{0}\right)\right) /\left(6 m_{\ell} t_{\text {ret }\left(K h_{2}\right)}\right)<0 \quad$ and $\left(\tau^{V}\left(\dot{\gamma}_{0}\right) t_{r e t\left(K h_{1}\right)}\right) /\left(120 m_{\ell} t_{\text {ret }\left(K h_{2}\right)}^{4}\right)<0$, where we use the equality $t_{\text {ret }(K)}=\mu / G, t_{\text {ret }\left(K h_{1}\right)}=m_{\ell} / \mu$, $t_{\text {ret }\left(K h_{2}\right)}=\left(m_{\ell} / G\right)^{1 / 2}$ and $\frac{t_{r e t(K)}}{t_{r e t\left(K h_{2}\right)}^{2}}=\frac{t_{r e t\left(K h_{1}\right)}}{t_{r e t\left(K h_{1}\right)} t_{r e t(K)}}=\frac{1}{t_{r e t(K)}}$.

Equation (7) in the plane $\alpha \rho^{2}$ is the equation of a parabola. From (7) it follows that:

$$
\rho^{2}=-\frac{3}{5} \frac{\beta}{\delta} \pm \sqrt{\frac{9}{25} \frac{\beta^{2}}{\delta^{2}}-\frac{8}{5} \frac{\alpha}{\delta}} .
$$

One root of the equation (7) for $\alpha=0$ is equal to zero, the second is negative, since $\beta$ and $\delta$ both of the same sign. For $\alpha>0$ the equation (7) we have only one positive root. Thus, the parabola will look as shown in Figure 2.

According to equation (6) the parabola

$$
\alpha+\frac{9}{4} \beta \rho^{2}+\frac{25}{8} \delta \rho^{4}=0
$$

determines the area of a plane $\alpha \rho^{2}$ of stability, where $\Phi^{\prime}(\rho)$ $<0$, from the instability of the region, where $\Phi^{\prime}(\rho)>0$. In this case, the stability of the region is located out of the parabola (8) shown in the Figure 2 with dashed curve. From Figure 2, it follows that when $\alpha<0$, it is only one stable equilibrium $\rho=0$. When $\alpha>0$ there are two states of equilibrium: unstable $\rho=0$ and stable, corresponding to the upper branch of the parabola.

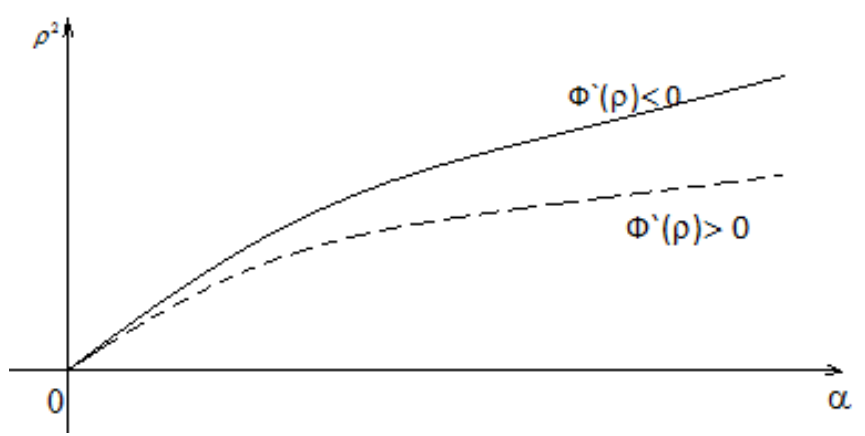

Fig. 2. Parabola of the equation (7) $\alpha=0$ as equal to zero $\Phi^{\prime}(\rho)<0$ and $\Phi^{\prime}(\rho)>0$.
When $\alpha<0$ in the phase plane $g \frac{d g}{d t}$ the stress-strain state of equilibrium is at the origin and, consequently, medium performs damping deformation vibrations. When $\alpha>0$, in the phase plane $g \frac{d g}{d t}$ an unstable equilibrium state is at the origin and a stable limited cycle (Figure 3 ).

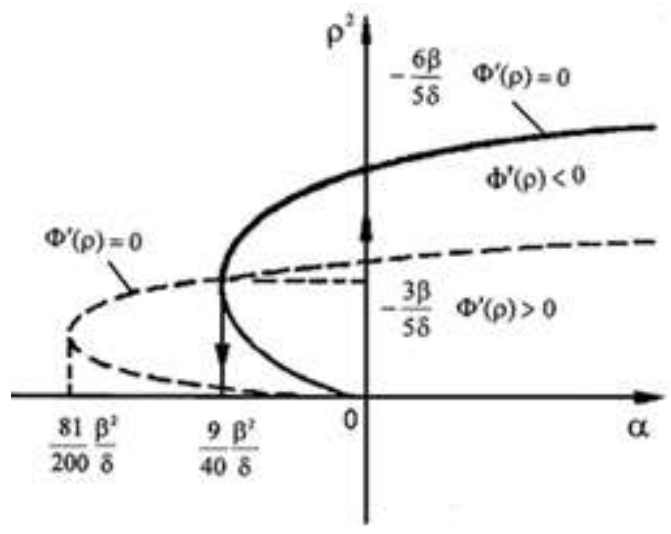

Fig. 3. Parabola of the equation (8) when $\alpha<0, \rho=0$ where $\Phi^{\prime}(\rho)>0$; when $\alpha>0$ where $\Phi^{\prime}(\rho)>0$.

When $\alpha$ is reduced the limit cycle shrinks to the origin, when $\alpha=0$ it merges with an unstable equilibrium state and gives it its stability. When $\alpha$ increases from negative to positive crossing zero self-oscillation, the amplitude of which increases continuously (with continuous increase of $\alpha$ ) occurs. This pattern of occurrence of oscillation in the deformation system is called mild excitation.

Consider the case $\beta>0, \delta<0$, i.e. $\left(\tau^{\prime \prime \prime}\left(\dot{\gamma}_{0}\right)\right) /\left(6 \mu t_{r e t\left(K h_{2}\right)}^{2}\right)>0$, and $\left(\tau^{(V)}\left(\dot{\gamma}_{0}\right)\right) /\left(120 \mu t_{r e t\left(K h_{2}\right)}^{4}\right)<0$, as $t_{r e t\left(K h_{2}\right)}$ and $\mu$, a are determined positive values, then it should be $\tau^{(V)}\left(\dot{\gamma}_{0}\right)<0$. In this case, the plane $\alpha \rho^{2}$ of the parabola (7) intersects the axis $\rho^{2}$ at the points $\alpha_{2}=0, \quad \rho_{2}^{2}=0$ and $\alpha_{3}=0$, $\rho_{3}^{2}=(-6 \beta) /(5 \delta)$. The location of the parabola is shown in Figure 4. In this case, the region of the deformation stability is out of the parabola (8), drawn by dashes in Figure 4. From Figure 4 it follows that when the medium parameters correspond to the following inequalities $\alpha<\left(9 \beta^{2}\right) /(40 \delta)$, i.e.

$$
-1+\left(\tau^{\prime}\left(\dot{\gamma}_{0}\right) t_{r e t\left(K h_{1}\right)}\right) / m_{\ell}<3\left(\tau^{\prime \prime \prime}\left(\dot{\gamma}_{0}\right)\right)^{2} /\left(4 \mu \tau^{V}\left(\dot{\gamma}_{0}\right)\right),
$$

then, there is only one stable equilibrium state of deformation and rheodynamcs system thus makes a damping vibration deformation.

When the parameters of the medium satisfy the following inequalities

$$
3\left(\tau^{\prime \prime \prime}\left(\dot{\gamma}_{0}\right)\right)^{2} /\left(4 \mu \tau^{V}\left(\dot{\gamma}_{0}\right)\right)<-1+\left(\tau^{\prime}\left(\dot{\gamma}_{0}\right) t_{r e t\left(K h_{1}\right)}\right) / m_{\ell}<0,
$$


then, the number of equilibrium states of deformation is equal to three: a stable state of equilibrium deformation $\rho=$ 0 , corresponding to the lower branch of the parabola (7) and a stable equilibrium deformation state corresponding to the top of the parabola (7). On the phase plane $g \frac{d g}{d t}$, this corresponds to a stable singular point at the origin, the unstable limit cycle and the stable limit cycle.

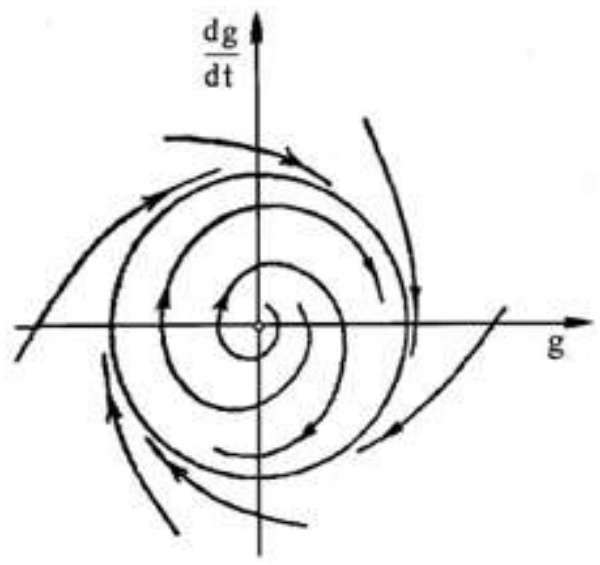

Fig. 4. Location of the parabola when $\alpha<\left(9 \beta^{2}\right) /(40 \delta)$.

\section{CONCLUSION}

Thus, for the initial conditions lying within the unstable limit cycle, the vibration deformations of rheodynamcs system are damping. With the initial conditions, located outside of this cycle, vibration deformation processes are established. When $\alpha>0$, the deformation state of equilibrium at the origin is unstable and for any initial conditions stable deformation self-oscillations are established (Figure 3). Note, if the rheological parameters of the system satisfy the following inequalities $\left(9 \beta^{2}\right) /(40 \delta)<\alpha<0$, i.e.

$$
3\left(\tau^{\prime \prime \prime}\left(\dot{\gamma}_{0}\right)\right)^{2} /\left(4 \mu \tau^{V}\left(\dot{\gamma}_{0}\right)\right)<-1+\left(\tau^{\prime}\left(\dot{\gamma}_{0}\right) t_{r e t\left(K h_{1}\right)}\right) / m_{\ell}<0,
$$

then the rheodynamcs system can be in equilibrium or selfoscillation deformation states. Therefore, if it is at rest, then, by giving it a quite large deformation rate, it can lead to a self-vibrating mode of deformation.

When we change $\alpha=-1+\left(\tau^{\prime}\left(\dot{\gamma}_{0}\right) t_{\text {ret }\left(K h_{1}\right)}\right) / m_{\ell}$ from negative to positive values, in rheodynamcs system self-vibrating deformation processes are occurred. Let at $\alpha<0$ the rheodynamcs system is in a stable non-deformed state, and at $\alpha=0$ self-oscillation deformations of finite amplitude are appeared. Further, by increasing $\alpha$, i.e. $\tau^{\prime}\left(\dot{\gamma}_{0}\right) t_{r e t\left(K h_{1}\right)}$, or decreasing $m_{\ell}$ the amplitude of the deformation vibrations increases. This mode of occurrence of the self-oscillation deformation is called "hard" mode of deformation.

In the reverse change of $\alpha$ - from positive to negative values - self-vibrating amplitude of deformation is constantly decreasing and at $\alpha=\left(9 \beta^{2}\right) /(40 \delta)$ self-vibrating deformation stops (at finite deformation amplitude) and the medium comes to a stable equilibrium state of deformation.

Parameter $\alpha$, resulting in different values to it the emergence and disappearance of self-oscillation in a medium, is called coefficient of excitation of rheodynamcs system.

Note that in the cases $\beta<0, \alpha>0$ and $\beta>0, \delta>0$ for certain values in rheodynamcs system can be infinitely increasing vibrational deformation. However, an unlimited increase in the vibration deformation requires unlimited admission of energy to the system. Therefore, in these cases, the approach taken under different $\tau\left(\dot{\gamma}_{0}+\dot{\gamma}\right)$ is enough and should take into account the higher-order terms.

\section{ACKNOWLEDGEMENTS}

The author would like to thank Prof. Ya.D. Khodzhaev, for support.

\section{REFERENCES}

[1] Eirich, F. R., ed., Rheology: Theory and Application, Vols. 1-5, Academic Press, Inc., New York, 19561970.

[2] Barnes, H.A., Hutton, J.E., and Walters K. (2000), A Handbook of Elementary Rheology. Aberystwyth: University of Wales.

[3] Butenin, N.V., Neumark, Y.I., and Fufaev, N.A. (1987) Introduction to the theory of nonlinear oscillations. Moscow, 384 p., (in Russian).

[4] Hoffman, J., and Johnson, C. (2000) Computational turbulent incompressible flow. Applied Mathematics: Body and Soul 4. Springer.

[5] Coleman, B. D., and Noll, W., (1961). Foundations of linear viscoelasticity.Review of Modern Physics, Vol. 33, No. 2, 239 - 249.

[6] Christova, I.C., and Christov C.I. (2016), Stress retardation versus stress relaxation in. Preprint submitted to Mechanics Research Communications. February 18, 2016. pp. 1-6.

[7] Dávalos-Orozco, L. A. (2012) Viscoelasticity - From Theory to Biological Applications. Chapter 1. Viscoelastic Natural Convection.

[8] Jeffreys, H. (1926). The stability of a layer of fluid heated from below. Philosophical Magazine Series 7, Vol. 2, No. 10, 833 - 844.

[9] Kapitaniak, T. (2000).Chaos for Engineers, SpringerVerlag, ISBN 3-540-66574-9, Berlin.

[10] Khusanov, I.N. (1990), Model of visco-elastic-inert deformable medium. In the book: The hydrodynamics of multiphase mediums and its applications. Tashkent, pp 139-159 (in Russian).

[11] Khusanov, I.N., and Mirzoev, A.A. (2015), Multiphase medium with complex rheology and their mechanical models. "XIth All-Russian Congress on Basic Problems of Theoretical and Applied Mechanics, August 20-24, 2015, Kazan, Russia” (in Russian).

[12] Kuehn., C. (2015), Multiple time scale dynamics. Springer. 
[13] Kuehn., C. (2015), The curse of instability. arXiv:1505.04334v1 [nlin. AO] 16 May 2015.

[14] Landau, L. D., and Lifshitz, E. M., (1987). Fluid Mechanics, Pergamon Press, ISBN 0-08-033933-6, New York.

[15] Larson, R.G. (1999), The Structure and Rheology of Complex Fluids, Oxford University Press, New York.

[16] Loitsyansky, L.G., (1987), Mechanika zidkosti i gaza (Fluid and gas mechanics), Moskva, Nauka.

[17] Lorenz, E. N. (1963). Deterministic non- periodic flows. Journal Atmospheric Science, Vol. 20, No. 2, pp. 130 - 141.

[18] Nigmatulin, R.I. (1990), Dynamics of Multiphase Media, Hemisphere, N.Y.

[19] Nigmatulin, R.I., Nigmatulin, B.I., Khodzhaev, Ya.D., and Kroshilin V.E.( 1996), Entrainment and deposition rates in a dispersed-film flow. Int. J. Multiphase Flow. Vol. 22, No 1, pp. 19 - 30

[20] Oldroyd, J. G. (1950). On the formulation of rheological equations of state.Proceedings of the Royal Society of London A, Vol. 200, No. 1063, 523 - 541.

[21] Schlichting, H. (1979). Boundary-layer theory, 7th ed. McGraw-Hill, New York.

[22] Seydametova, Z.S. (1990), Investigation of rheology of the medium depending on the frequency and amplitude of the current stress. In the book: Hydrodynamics of multiphase media and its applications. Tashkent, pp 160 -173 (in Russian)

[23] Seydel, R. (2010), Practical bifurcation and stability analysis. Springer, Third Edition.

[24] Wiggins, S. (2003), Introduction to applied nonlinear dynamical systems and chaos. New York, Second Edition. Springer. 